\title{
Application of SVM Algorithm for Particle Swarm Optimization in Apple Image Segmentation
}

\author{
Qirui Huang \\ School of Electronic and Electrical Engineering, \\ Nanyang Institute of Technology, Nanyang 473004, China. \\ qirui0607@gmail.com
}

Keywords: image segmentation; machine vision; particle swarm algorithm; parameter optimization; SVM

\begin{abstract}
The apple image segmentation is the key technology of identification and location in the apple-picking machine vision system. On account of huge errors in the process of discriminating fruits by apple-picking robots at present and the long-time processing, the SVM theory in fingerprint image segmentation method is conducted. Combined with the global search ability of particle swarm optimization in solving combinational optimization problems, the SVM partitioning algorithm, which is based on the parameter optimization of particle swarm, is put forward. The results show that this algorithm makes the separation of apple fruits and the image background come true. It also preserves the outline of apples, then polishes the image after segmentation by the close operation in mathematical morphology, which eliminates the pore phenomenon effectively and provides convenience for the further apple-picking and apple-discriminating.
\end{abstract}

\section{Introduction}

The apple-picking robot mainly has two tasks: one is to discriminate and locate apples accurately, the other is to pick the fruits losslessly[1]. The primary task is to discriminate and locate apples by the machine vision system. The key point in this task is image processing. To solve this problem, it is necessary to abstract the images of apple fruits from the complicated background images, namely the segmentation of the actual shooting of color image. Now there are many common algorithms used in image segmentation, such as: thresholding method[2], region growing method[3], 2D histogram for edge detection and boundary tracking method[4] and so on. Due to the complexity of the environment of apple-picking, especially the uncertainty of light condition and the partial and all occlusion problem of fruits, the recognition rate of fruits is not high. So the intelligent discriminating and location of the picking objects need further research.

SVM is a kind of machine learning based on the statistical learning theory, which has the good ability of generalization and is widely used in the field of pattern recognition, such as image classification and so on[5]. Compared with algorithms which concentrate on the minimum empirical risk, SVM could has a more accurate generalization, and has the advantage in the recognition of nonlinear model. Practices have proved that the parameter of kernel function of SVM and the penalty coefficient have great influence on the classification performances of SVM[6]. In terms of some systems which have a higher real-time demand, how to choose the proper SVM parameter quickly and reduce the time of training and discriminating has very significant practical meaning. So the thesis puts forward the SVM sorting algorithm based on the PSO. By using the PSO to seek the optimal parameter of SVM, the purpose of optimizing the disaggregated model can be achieved, and the model can be used in the segmentation of the images of apple fruits. The results show that the algorithm could apparently improve the segmentation of the images of apple fruits. 


\section{The priciple of Support Vector Machine}

SVM is the newly concept and the most useful part in the statistical learning theory. SVM takes training error as the constraint condition to optimization problem and takes minimizing the confidence interval values as optimization objective[7]. The basic principle is to find a segmentation plane which meets the requirement, so that the point of the training set is far away from the plane as much as possible.

The non-linear modeling based on support vector machines is concretely delivered as follows:

Given a dataset $\left(x_{i}, y_{i}\right), i=1, \cdots, N, \boldsymbol{x}_{i} \in \boldsymbol{R}^{d}$ belonging to two separate classes where $y_{i} \in\{+1,-1\}$. If there is a classification plane, so that the sum of the shortest distance between the classification plane and the two classes, and the shortest distance from the classification plane to support vector of each class are equal, this classification plane is called optimal classification plane. It can classify all training vectors correctly and the vectors of each class are separated with a maximum margin. A quadratic optimization that can solve this problem is formulated as follow:

$$
\left\{\begin{array}{l}
\min _{w . b . \xi_{i}} \frac{1}{2}\|W\|^{2}+C \sum_{i}^{n} \xi_{i} \\
\text { s.t. } \quad y_{i}\left[\left(w \cdot x_{i}\right)+b\right] \geq 1-\xi_{i}, \xi_{i} \geq 0, i=1,2, \cdots, n
\end{array}\right.
$$

The boundary of different type of data is defined by vector $W, b$ is a scalar threshold, $C$ is the penalty parameter, it can control the training error rate by different values. For a non -linearly problem, equation can be converted to

$$
\left\{\begin{aligned}
\min _{a} & \frac{1}{2} \sum_{i=1}^{n} \sum_{j=1}^{n} \alpha_{i} \alpha_{j} y_{i} y_{j} \exp \left(-\gamma\left\|x_{i}-x_{j}\right\|^{2}\right)-\sum_{i=1}^{n} \alpha_{i} \\
& (i=1,2, \cdots, n) \\
\text { s.t. } & \sum_{i=1}^{n} \alpha_{i} y_{i}=0,0 \leq \alpha_{i} \leq C
\end{aligned}\right.
$$

Where $K\left(x_{i}, x_{j}\right)=\exp \left(-\gamma\left\|x_{i}-x_{j}\right\|^{2}\right)$ is the kernel function which is the radial basis function (RBF), it can change the input data to a higher dimensional space, and change a non-linearly separable problem in low dimension to a linearly separable problem.

The equation (2) shows that the result of the minimization is determined by the selection of the parameters $(C, \gamma)$. So this article SVM parameter optimization, generally $(C, \gamma)$ as the pending optimization variables.

\section{The principle of Particle Swarm Optimization}

PSO is a swarm intelligence algorithm which was put forward by Eberhart and Kennedy in 1995. It performs the global search through collaboration and competition between individuals stemming from the predatory behavior, and is used to solve searching optimization results[8]. PSO has the advantages of fast execution, and affected by the problem of dimension changes small, it's described as follows:

First, generate a group of particles in the feasible solution space randomly, each particle is a potential solution of optimization problems, and there is an objective function to determine the fitness(Fitness Value) corresponding to. Each particle flights in the solution space, it's direction and distance is determined by a speed. During each iteration, the particle will follow the two extremes: One is individual extreme which is found the optimal solution in the particle itself so far, and the other is global extreme which is found in the population so far. After finding the two extreme values, particles will update their velocity and position according to the following formula[9]: 


$$
\begin{aligned}
& v_{i d}^{t+1}=w^{t} \cdot v_{i d}^{t}+c_{1} \cdot r_{1} \cdot\left(p_{i d}^{t}-x_{i d}^{t}\right)+c_{2} \cdot r_{2} \cdot\left(p_{g d}^{t}-x_{i d}^{t}\right) \\
& x_{i d}^{t+1}=x_{i d}^{t}+v_{i d}^{t+1}
\end{aligned}
$$

where $t$ is the evolving generation; $c_{1}$ and $c_{2}$ denote personal and social learning factors, whose values are positive and generally assigned to $2 ; r_{1}$ and $r_{2}$ are random variables generated from a uniform distribution in the range [0,1]; Termed the "inertia weight", $w$ is introduced to compromise and balance the global exploration and local exploitation; $x_{i d}$ represents the position of particle $i$ on dimension $d$; and $v_{i d}$ means the velocity of particle $i$ on dimension $d$.

\section{The algorithm of PSO-SVM}

The paper uses PSO to find the best values of $\mathrm{c}$ and $\mathrm{g}$ of SVM. The algorithm is as follows[10]:

1. Initialize parameters, particle populations, the position and velocity of particle and so on.

2. Calculate individual fitness value $\left(f_{i}\right)$ of particle, each particle will compare $f_{i}$ with the best fitness $\left(p_{i d}\right)$ which calculates from the experienced position. update its $p_{i d}$ if $f_{i}$ is better.

3. compare $p_{i d}$ with the fitness of global optimum $p_{g d}$, if better, update its $p_{g d}$.

4. Modify the velocity and position of particle according to Formula 3.

5. If satisfy the set conditions, stop optimization; otherwise go to step 2.

6. Get $p_{g d}$ from 5, the SVM parameters $\gamma$ and values of $C$ are obtained.Train the SVM until the result is satisfied.

\section{Results and Discussion}

The platform of this software in the test is MATLAB R2011a. The main target of the test is the images of apples with real-time shooting which have a complicated background under natural conditions. There are totally 1000 training samples including 500 samples of fruits regions and 500 samples of background regions. Then people put there training samples into the SVM to train, then search the best parameter in SVM combined with PSO mentioned in the thesis. Finally people use the best parameter that they get to construct the SVM classification model. Then they use the SVM classification model to make the segmentation of images to get the images of apple fruits that have already been divided.In order to achieve better results, there should be a dilation operation of the images after segmentation to achieve the ideal results, which could meet the post-processing demands of images.

In order to testify the segmentation accuracy of the algorithm, the region of the fruits that is divided by Photoshop is shown in figure 1(e). It can be seen from figure 1(d) that the segmentation images of the fruits which are made by the means in the thesis have a smooth edge, and the region of the segmentation images of the fruits is similar to the region of actual region of fruits. To have a objective judgment of this algorithm, according to the evaluation method mentioned by George, people could evaluate the segmentation results by the detection rate and false detection rate.

$$
\begin{aligned}
\text { Detection rate } & =\left(B W \cap B W_{\text {real }}\right) / B W_{\text {real }} \\
\text { False detection rate } & =\left(B W-B W \cap B W_{\text {real }}\right) / B W_{\text {real }}
\end{aligned}
$$

$B W_{\text {real }}$ is the region of fruits picked by people, and $B W$ is the region of fruits divided by the algorithm in the thesis. As shown in table 1, people could get the detection rate and false detection rate of the results of segmentation according to the this method. The results shown in table 1 reveal that the detection rate of the three image samples is over $90 \%$. The reason why Sample 1 has lower detection rate is that the apple is covered by branches. This makes one of the apple images loss a small part, which is the background in the post-processing. It is obvious in the table that the detection rates 
of the other two images are over $99.05 \%$ and $97.08 \%$. The false detection rate of the three images is lower than $2.0 \%$, which could achieve a better segmentation result.

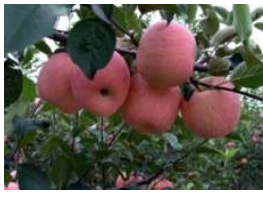

(a1)sample 1

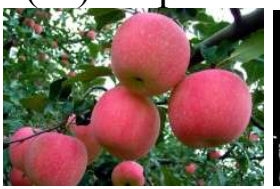

(a2)sample 1

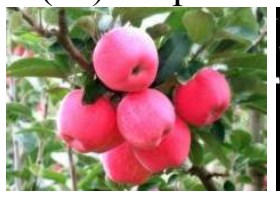

(a3)sample 1

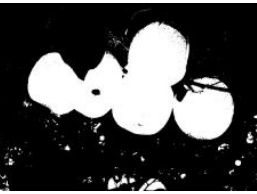

(b1) initial

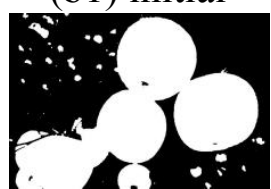

(b2) initial

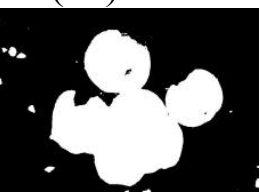

(b3) initial

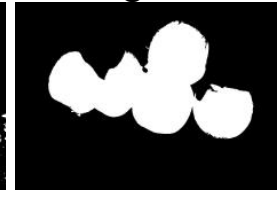

(c1) Post-proces
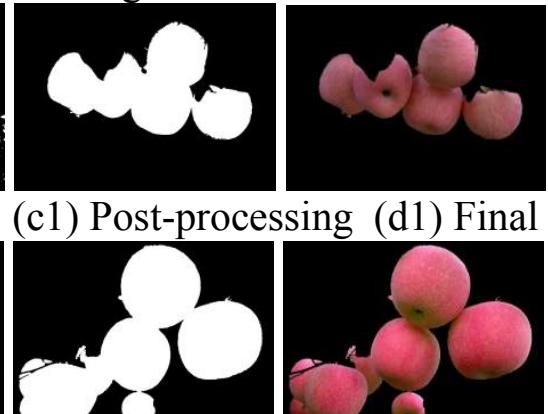

(c2) Post-process

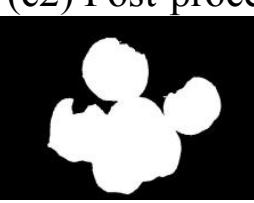

(c3) Post-processing
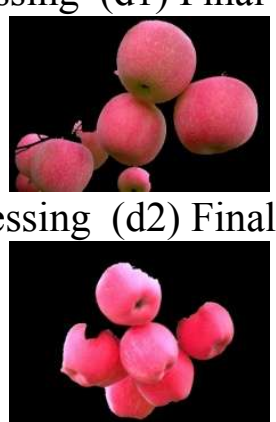

d3) Final

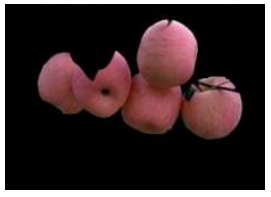

(e1) manual

Figure 1 results of Segmentation

Table 1 Results of the Segmentation of Apple Images

\begin{tabular}{lll}
\hline Sample & Detection rate/\% & False detection rate/\% \\
\hline Sample 1 & 92.81 & 0.67 \\
Sample 2 & 99.05 & 1.53 \\
Sample 3 & 97.08 & 2.00 \\
\hline
\end{tabular}

\section{Summary}

The paper uses the SVM segmentation algorithm based on PSO to divide the apple images, which separates the ripe apple fruits with the background well. In the following process, with the post-processing of erosion, dilation and other operations, the outline of the apple could be preserved to provide the necessary condition for further discriminating and location of apple-picking machines.

\section{References}

[1] Bulanon D M, Kataoka T, Okamoto H, et al. Development of a real-time machine vision system for the apple harvesting robot[J]. Sice Conference, 2004, 1:595 - 598.

[2] Zhao M, Lin H Y, Yang C H, et al. Automatic threshold level set model applied on MRI image segmentation of brain tissue[J]. Appl. Math, 2015, 9(4): 1971-1980.

[3] Tianhui S, Bugong X, Zhanhu H. Medical image segmentation based on region growing arithmetic[J]. Microcomputer Information, 2008, 24(6): 284-286.

[4] Choi J W, Whangbo T K, Kim C G. A contour tracking method of large motion object using optical flow and active contour model[J]. Multimedia Tools and Applications, 2015, 74(1): 199-210. [5] VAPNIK V, LEVIN E, CUN Y L. Measuring the VC-dimension of learning machines[J]. Neural Computation, 1994, 6(5): 851-876

[6] CHAPELLE O, VAPNIK V, BOUSQUET O. Choosing multiple parameters for support vector machines[J]. Machine Learning, 2002, 46(1/3): 131-159.

[7] Fasshauer G E, Hickernell F J, Ye Q. Solving support vector machines in reproducing kernel Banach spaces with positive definite functions $[\mathrm{J}]$. Applied and Computational Harmonic Analysis, 2015, 38(1): 115-139. 
[8] Fister I, Perc M, Ljubič K, et al. Particle swarm optimization for automatic creation of complex graphic characters[J]. Chaos, Solitons \& Fractals, 2015, 73: 29-35.

[9] Pornsing C, Sodhi M S, Lamond B F. Novel self-adaptive particle swarm optimization methods[J]. Soft Computing, 2015: 1-15.

[10] Xiaomei Y, Peng W, Lijuan L. A Study of Effective Irrigation Area Forecast Model Based on PSO-SVM[J]. AISS: Advances in Information Sciences and Service Sciences, 3(9): 360-367. 